\title{
Asymptotic behaviour of the poles of a special generating function for acyclic digraphs
}

\author{
Peter Grabner and Bertran Steinsky
}

Institut für Analysis und Computational Number Theory (Math A) 


\title{
ASYMPTOTIC BEHAVIOUR OF THE POLES OF A SPECIAL GENERATING FUNCTION FOR ACYCLIC DIGRAPHS
}

\author{
PETER J. GRABNER AND BERTRAN STEINSKY
}

\begin{abstract}
Let $z_{k}$ be the $k$-th zero of $\phi(z)=\sum_{n=0}^{\infty} \frac{(-z)^{n}}{n ! 2\left(\begin{array}{c}n \\ 2\end{array}\right)}$ sorted increasingly by modulus from the origin, for $k \geq 0$. $\frac{1}{\phi}$ appears as special generating function for acyclic digraphs and $\phi$ satisfies the functional differential equation $\phi^{\prime}(z)=-\phi\left(\frac{z}{2}\right)$. It is a conjecture of R.W. Robinson that $z_{k}=(k+1) 2^{k}+o\left(2^{k}\right)$. We show that there is a $K$ such that $z_{K+k}=(k+1) 2^{k}+o\left(\frac{2^{k}}{(k+1)^{1-\epsilon}}\right)$ for all $\epsilon>0$.
\end{abstract}

\section{INTRODUCTION}

Acyclic digraphs have been studied by several authors, e.g., Robinson [3],[4] and Stanley [5]. Let

$$
S(z)=\sum_{n=0}^{\infty} \frac{s_{n} z^{n}}{n !} .
$$

Robinson defined $\Delta$ in [3] to be the linear operation on exponential generating functions which divides $x^{n}$ by $2^{\left(\begin{array}{c}n \\ 2\end{array}\right)}$, i.e.,

$$
\Delta S(z)=\sum_{n=0}^{\infty} \frac{s_{n} z^{n}}{n ! 2^{\left(\begin{array}{c}
n \\
2
\end{array}\right)} .}
$$

He called $\Delta S(z)$ the special generating function for $S$, which he used, for example, to count labelled directed acyclic graphs in [3]. Let $a_{n}$ be the number of labelled directed acyclic graphs with $n$ vertices and

$$
A(z)=\sum_{n=0}^{\infty} \frac{a_{n} z^{n}}{n !} .
$$

In this paper we will analyse the zeros of the function

$$
\Delta e^{-z}=\sum_{n=0}^{\infty} \frac{(-z)^{n}}{n ! 2^{\left(\begin{array}{c}
n \\
2
\end{array}\right)}}
$$

Date: February 14, 2008.

1991 Mathematics Subject Classification. Primary: 05A16 Secondary: 39B32, 30D05, 34K25, 30E15, 32A60.

Key words and phrases. Functional differential equation, special generating function, labelled directed acyclic graph, zero, asymptotic behaviour, saddle point method.

The authors are supported by the START-project Y96-MAT of the Austrian Science Fund. 
which we denote by $\phi(z)$. Robinson observed that $\phi(z)$ satisfies the functional differential equation

$$
\phi^{\prime}(z)=-\phi\left(\frac{z}{2}\right)
$$

and that

$$
\left(\Delta e^{-z}\right)(\Delta A(z))=1 .
$$

Figure 1, which we produced with Mathematica, shows a plot of this function. Let $z_{k}$ be

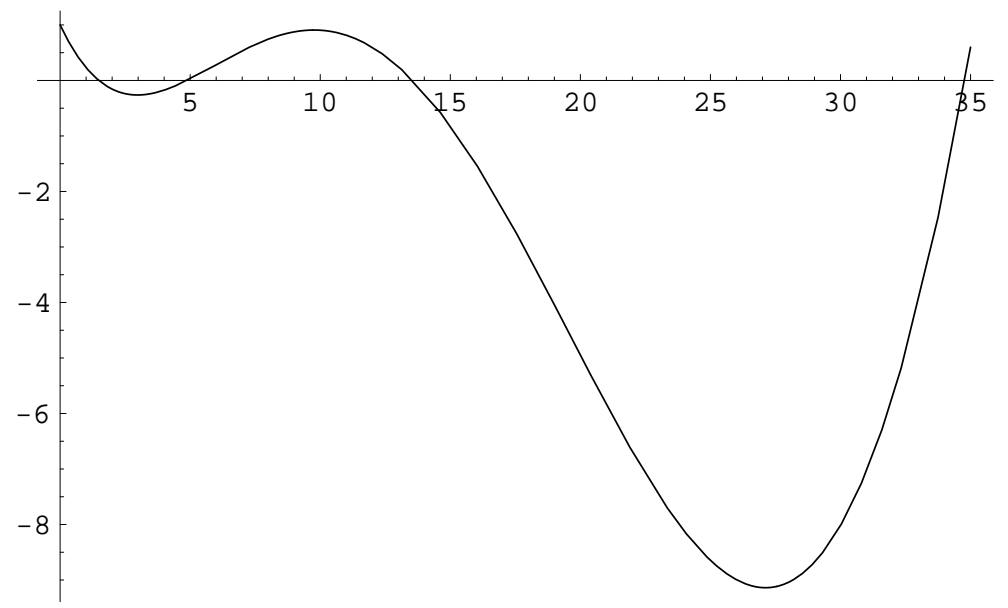

FiguRE 1. $\phi(z)$

the $k$-th zero of $\phi(z)$ sorted increasingly by modulus from the origin for $k \geq 0$. Robinson proved that all zeros of $\phi(z)$ are simple, positive real and satisfy $z_{k+1}>2 z_{k}$ for $k \geq 0$. Furthermore he conjectured that $z_{k}=(k+1) 2^{k}+o\left(2^{k}\right)$ in [3]. We prove a slightly different result, namely Theorem 2 and present an asymptotic representation of the function $\phi$ in Theorem 1. Table 1 gives a comparison between the $k$-th zero $z_{k}$ and $(k+1) 2^{k}$.

\section{The Zeros of $\phi(z)$}

First we state

Lemma 1. The function $\phi(z)$ can be written as

$$
\phi(z)=\frac{1}{2 \pi i} \int_{\mathcal{H}} \frac{\pi}{\sin (\pi s)} \frac{1}{\Gamma(1-s) 2^{\frac{s(s+1)}{2}} z^{s}} d s,
$$

where the Hankel-contour $\mathcal{H}$ encircles the negative integers and 0.

Proof. We observe

$$
\phi(z)=\sum_{n=0}^{\infty} \frac{(-z)^{n}}{n ! 2^{\left(\begin{array}{c}
n \\
2
\end{array}\right)}}=\frac{1}{2 \pi i} \int_{\mathcal{H}} \frac{\Gamma(s)}{2^{\frac{s(s+1)}{2}} z^{s}} d s,
$$




\begin{tabular}{|l|l|l|}
\hline$k$ & $z_{k}$ & $2^{-k} z_{k}-(k+1)$ \\
\hline 0 & $1.48807854559971029465 \ldots$ & 0.488079 \\
1 & $4.88114089489665468076 \ldots$ & 0.44057 \\
2 & $13.560408527963422630046 \ldots$ & 0.390102 \\
3 & $34.775316247750907131517 \ldots$ & 0.346915 \\
4 & $84.977290107207686550531 \ldots$ & 0.311081 \\
5 & $201.002876160804469905966 \ldots$ & 0.28134 \\
6 & $464.412828169898831201845 \ldots$ & 0.25645 \\
7 & $1054.131896685496993769517 \ldots$ & 0.235405 \\
8 & $2359.661041195481673546868 \ldots$ & 0.217426 \\
9 & $5223.380439502564290102436 \ldots$ & 0.201915 \\
10 & $11456.93506311994880902310 \ldots$ & 0.188413 \\
11 & $24937.60380074991930495522 \ldots$ & 0.176564 \\
12 & $53928.30177867352491973944 \ldots$ & 0.166089 \\
13 & $115972.233276771948755379770 \ldots$ & 0.156767 \\
14 & $248191.706915839202599726065 \ldots$ & 0.14842 \\
15 & $528905.163307529387654815292 \ldots$ & 0.140905 \\
16 & $1122900.700985837445143423214 \ldots$ & 0.134105 \\
17 & $2376063.281403420485104790828 \ldots$ & 0.127924 \\
18 & $5012791.625019875403953253170 \ldots$ & 0.122283 \\
19 & $10547160.909492859829473254757 \ldots$ & 0.117113 \\
20 & $22137913.099346041141374364701 \ldots$ & 0.112359 \\
21 & $46363780.401215272096183164933 \ldots$ & 0.107973 \\
22 & $96904841.306209099621461578720 \ldots$ & 0.103915 \\
23 & $202166693.910570234291360571678 \ldots$ & 0.100148 \\
24 & $421051803.671740123329889100282 \ldots$ & 0.0966432 \\
25 & $875548345.417406748367314625259 \ldots$ & 0.0933741 \\
\hline
\end{tabular}

TABLE 1. The zeros $z_{k}$ compared to $(k+1) 2^{k}$.

since

$$
\operatorname{Res}\left(\Gamma(s) 2^{-\frac{s(s+1)}{2}} z^{-s},-n\right)=\frac{(-1)^{n}}{n !} 2^{-\frac{n(n-1)}{2}} z^{n}, \text { for } n \geq 0,
$$

and Stirling's formula guarantees that the integral converges. The result follows with the functional equation $\Gamma(s) \Gamma(1-s)=\frac{\pi}{\sin (\pi s)}$.

We notice that the saddle point method does not work if we use (1), since there are oscillating terms, as we will see later.

We rewrite $\phi(z)$ with

$$
w(s, t)=-\log \Gamma(1-s)-\frac{s(s+1)}{2} \log 2-s t
$$


in the form

$$
\phi(z)=\frac{1}{2 \pi i} \int_{\mathcal{H}} \frac{\pi}{\sin (\pi s)} e^{w(s, \log z)} d s,
$$

where log, in the whole paper, means the principal value of the complex logarithm. To simplify the following notation we write $x=\log z$ and

$$
\tilde{\phi}(x)=\frac{1}{2 \pi i} \int_{\mathcal{H}} \frac{\pi}{\sin (\pi s)} e^{w(s, x)} d s .
$$

Since we will use the saddle point method, which is discussed for example in [1] or [2], in a slightly modified way, we are now interested in zeros of $\partial w(s, x) / \partial s$. First, we rewrite

$$
\begin{aligned}
w(s, x) & =-\log \Gamma(1-s)-\frac{s(s+1)}{2} \log 2-s x \\
& =-\log ((-s) \Gamma(-s))-\frac{s(s+1)}{2} \log 2-s x \\
& =-\log (-s)-\log \Gamma(-s)-\frac{s(s+1)}{2} \log 2-s x
\end{aligned}
$$

at least if $\operatorname{Re} s<0$, and it will turn out that this is all we need. Therefore, we search for solutions of

$$
-\frac{1}{s}+\psi(-s)-\left(s+\frac{1}{2}\right) \log 2-x=0,
$$

where $\psi(s)$ is the first derivative of $\log \Gamma(s)$. Let $\sigma(x)$ denote the solution of $(2)$. Then the following asymptotic expansion holds.

We remark that throughout the whole article all error terms are considered as $x$ goes to infinity.

Lemma 2. The solution $\sigma(x)$ of the equation (2) satisfies

$$
\begin{aligned}
& \sigma(x)=\frac{1}{\log 2}\left(-x+\log x+C_{1}+\frac{\log x}{x}+\frac{C_{2}}{x}\right)+O\left(\frac{\log ^{2} x}{x^{2}}\right), \text { where } \\
& C_{1}=-\frac{1}{2}-\frac{\log \log 2}{2} \quad \text { and } C_{2}=\frac{3}{2} \log 2+\log \log 2 .
\end{aligned}
$$

Proof. We find the constants in

$$
\sigma(x)=A x+B \log x+C+\frac{D \log x}{x}+\frac{E}{x}
$$

by inserting $\sigma(x)$ into (2). First, we use the asymptotic expansion

$$
\psi(s)=\log s-\frac{1}{2 s}+O\left(\frac{1}{s^{2}}\right)
$$

to obtain

$$
\log (-\sigma(x))-\frac{1}{2 \sigma(x)}+O\left(\frac{1}{\sigma(x)^{2}}\right)-\left(\sigma(x)+\frac{1}{2}\right) \log 2-x=0
$$


from (2). Now, we have on the one hand

$$
\begin{aligned}
& \log \left(A x+B \log x+C+\frac{D \log x}{x}+\frac{E}{x}\right) \\
& =\log (A x)+\frac{B \log x+C+\frac{D \log x}{x}+\frac{E}{x}}{A x} \\
& +O\left(\left(\frac{B \log x+C+\frac{D \log x}{x}+\frac{E}{x}}{A x}\right)^{2}\right) \\
& =\log (A x)+\frac{B \log x+C+\frac{D \log x}{x}+\frac{E}{x}}{A x}+O\left(\left(\frac{\log x}{x}\right)^{2}\right),
\end{aligned}
$$

and on the other hand

$$
\begin{aligned}
& \frac{1}{2\left(A x+B \log x+C+\frac{D \log x}{x}+\frac{E}{x}\right)} \\
& =\frac{1}{2 A x}+O\left(\frac{B \log x+C+\frac{D \log x}{x}+\frac{E}{x}}{(A x)^{2}}\right)=\frac{1}{2 A x}+O\left(\frac{\log x}{x^{2}}\right) .
\end{aligned}
$$

The calculation of the constants is now straight forward.

Lemma 3. Let $0<\alpha<\frac{1}{3}, \beta=2-3 \alpha, \sigma=\sigma(x),|t|<x^{\alpha}$, and $h(x)=\frac{\log 2}{2}-\frac{1}{2(A x+B \log x)}$. Then

$$
w(\sigma+t, x)=w(\sigma, x)-h(x) t^{2}+O\left(\frac{1}{x^{\beta}}\right) .
$$

Proof. It is more convenient to write

$$
w(s, x)=g(s)-x s \text { where } g(s)=-\log (-s)+\log \Gamma(-s)-\frac{s(s+1)}{2} \log 2 .
$$

By Taylor expansion around $\sigma$ we have

$$
\begin{aligned}
w(\sigma+t, x) & =g(\sigma+t)-x(\sigma+t) \\
& =g(\sigma)-x \sigma+\frac{1}{2} g^{\prime \prime}(\sigma) t^{2} \\
& +t^{3}\left(\frac{1}{6} g^{\prime \prime \prime}(\sigma)+\frac{1}{24} g^{\prime \prime \prime \prime}(\sigma) t+\ldots\right) \\
& =w(\sigma, x)+\left(-\frac{1}{\sigma}+\psi(-\sigma)-\left(\sigma+\frac{1}{2}\right) \log 2-x\right) t \\
& +\frac{1}{2}\left(\frac{1}{\sigma^{2}}-\psi^{\prime}(-\sigma)-\log 2\right) t^{2} \\
& +t^{3}\left(\frac{1}{6} g^{\prime \prime \prime}(\sigma)+\frac{1}{24} g^{\prime \prime \prime \prime}(\sigma) t+\ldots\right)
\end{aligned}
$$


Since $\psi^{(n)}(\sigma)=O\left(1 / \sigma^{n}\right)$, for $n \geq 1$, we have $g^{(n+1)}(\sigma)=O\left(1 / \sigma^{n}\right)$ and therefore

$$
t^{3}\left(\frac{1}{6} g^{\prime \prime \prime}(\sigma)+\frac{1}{24} g^{\prime \prime \prime \prime}(\sigma) t+\ldots\right)=O\left(\frac{t^{3}}{\sigma^{2}}\right) .
$$

Now, we use Lemma $2, \psi^{\prime}(\sigma)=1 / \sigma+O\left(1 / \sigma^{2}\right)$, and $\psi^{\prime \prime}(\sigma)=-1 / \sigma^{2}+O\left(1 / \sigma^{3}\right)$ to obtain

$$
w(\sigma+t, x)=w(\sigma, x)+\left(-\frac{\log 2}{2}+\frac{1}{2 \sigma}+O\left(\frac{1}{\sigma^{2}}\right)\right) t^{2}+O\left(\frac{t^{3}}{\sigma^{2}}\right) .
$$

We use $|t| \leq x^{\alpha}$ to estimate the error term

$$
O\left(\frac{t^{3}}{\sigma^{2}}\right)=O\left(\frac{1}{x^{2-3 \alpha}}\right)
$$

If we expand $\sigma=A x+B \log x+O(1)$ we have

$$
\left(\frac{1}{2 \sigma}+O\left(\frac{1}{\sigma^{2}}\right)\right) t^{2}=\frac{t^{2}}{2(A x+B \log x)}+O\left(\frac{1}{x^{2-2 \alpha}}\right) .
$$

Therefore, $1<\beta=2-3 \alpha<2-2 \alpha<2-\alpha<2$.

Lemma 4. The real part of $w(\sigma(x) \pm i+t, x)$ is

$$
\begin{aligned}
& \frac{x^{2}}{2 \log 2}-\frac{x \log x}{\log 2}+x\left(\frac{1}{2}+\frac{1}{\log 2}-\frac{\log \log 2}{\log 2}\right) \\
& -t^{2}\left(\frac{\log 2}{2}+\frac{\log 2}{x}\right)+t O\left(\frac{\log x}{x}\right)+O\left((\log x)^{2}\right) .
\end{aligned}
$$

Proof. We use Stirling's formula to gain the asymptotic expansion

$$
w(s, x)=\left(s-\frac{1}{2}\right) \log s-s+\frac{\log (2 \pi)}{2}+\frac{1}{12 s}-\frac{s(s+1)}{2} \log 2-x s+O\left(\frac{1}{s^{3}}\right) .
$$

Using Lemma 2 and (3), we obtain $w(\sigma+t, x)$, a rather lengthy expression, we omit here. Similar as in the proof of Lemma 2, we substitute in this expression

$$
\begin{aligned}
& \log (A x)+\frac{B \log x+C+\frac{D \log x}{x}+\frac{E}{x}+t}{A x} \\
& -\frac{1}{2}\left(\frac{B \log x+C+\frac{D \log x}{x}+\frac{E}{x}+t}{A x}\right)^{2}+O\left(\left(\frac{\log x}{x}\right)^{3}\right)
\end{aligned}
$$

for $\log (\sigma+t)$ and

$$
\frac{1}{A x}+O\left(\frac{\log x}{x^{2}}\right)
$$

for $1 /(\sigma+t)$. We use Mathematica for the algebraic simplifications. 
Now, we let the curves $\mathcal{C}_{1}(t)=\sigma(x)+t$ and $\mathcal{C}_{2}(t)=\sigma(x)-t$, for $t \in\left[-x^{\alpha}, x^{\alpha}\right]$, where $0<\alpha<\frac{1}{3}$ and

$$
\tilde{\phi}_{\mathcal{C}_{1 / 2}}(x)=\frac{1}{2 \pi i} \int_{\mathcal{C}_{1 / 2}} \frac{\pi}{\sin (\pi s)} e^{w(s, x)} d s .
$$

Lemma 5.

$$
\tilde{\phi}(x)=\tilde{\phi}_{\mathcal{C}_{1}}(x)+\tilde{\phi}_{\mathcal{C}_{2}}(x)+e^{w(\sigma(x), x)} O\left(\frac{1}{x^{\beta}}\right) .
$$

Proof. We define the Hankel contour $\mathcal{H}$ in Lemma 1 to consist of three curves $H_{1}, H_{2}$, and $H_{3}$, where $H_{1}(t)=t+i$ for $t \in(-\infty, 1], H_{2}(t)=1-i t$ for $t \in(-1,1)$ and $H_{3}(t)=-t-i$ for $t \in(-1, \infty]$. Therefore, $\mathcal{H}$ circulates the negative integers and 0 . We abbreviate

$$
s(t)=\frac{\pi}{\sin (\pi(\sigma+t+i))},
$$

and estimate

$$
\begin{aligned}
& \frac{1}{2 \pi}\left|\int_{-\infty}^{-x^{\alpha}} \frac{\pi}{\sin (\pi(\sigma+t+i))} e^{w(\sigma+t+i, x)} d t-\int_{-\infty}^{-x^{\alpha}} \frac{\pi}{\sin (\pi(\sigma+t-i))} e^{w(\sigma+t-i, x)} d t\right| \\
& =\frac{1}{2 \pi}\left|\int_{-\infty}^{-x^{\alpha}} e^{\operatorname{Re} w(\sigma+t+i, x)}\left(s(t) e^{i \operatorname{Im} w(\sigma+t+i, x)}-\overline{s(t)} e^{-i \operatorname{Im} w(\sigma+t+i, x)}\right) d t\right| \\
& =\frac{1}{2 \pi}\left|\int_{-\infty}^{-x^{\alpha}} e^{\operatorname{Re} w(\sigma+t+i, x)} \operatorname{Re}(s(t)) 2 i \sin (\operatorname{Im} w(\sigma+t+i, x)) d t\right| \\
& +\frac{1}{2 \pi}\left|\int_{-\infty}^{-x^{\alpha}} e^{\operatorname{Re} w(\sigma+t+i, x)} \operatorname{Im}(s(t)) 2 \cos (\operatorname{Im} w(\sigma+t+i, x)) d t\right| \\
& \leq c_{1} \int_{-\infty}^{-x^{\alpha}} e^{\operatorname{Re} w(\sigma+t+i, x)} d t
\end{aligned}
$$

for a positive constant $c_{1}$. Now, we use Lemma 4 to continue with

$$
\begin{aligned}
& \int_{-\infty}^{-x^{\alpha}} e^{\operatorname{Re} w(\sigma+t+i, x)} d t \\
& =e^{\operatorname{Re} w(\sigma+i, x)+O\left((\log x)^{2}\right)} \int_{-\infty}^{-x^{\alpha}} e^{-t^{2}\left(\frac{\log 2}{2}+\frac{\log 2}{x}\right)+t O\left(\frac{\log x}{x}\right)} d t \\
& \leq e^{\operatorname{Re} w(\sigma+i, x)+O\left((\log x)^{2}\right)} \int_{-\infty}^{-x^{\alpha}} e^{-c_{2} t x^{\alpha}} \leq \frac{1}{c_{2} x^{\alpha}} e^{\operatorname{Re} w(\sigma+i, x)+O\left((\log x)^{2}\right)} e^{-c_{2} x^{2 \alpha}}
\end{aligned}
$$

for a positive constant $c_{2}$. Lemma 3 yields $w(\sigma+i, x)=w(\sigma, x)+h(x)+O\left(\frac{1}{x^{\beta}}\right)=$ $w(\sigma, x)+O\left(\frac{1}{x}\right)$. Therefore,

$$
e^{\operatorname{Re} w(\sigma+i, x)+O\left((\log x)^{2}\right)} e^{-c_{2} x^{\alpha}}=e^{w(\sigma, x)} O\left(\frac{1}{x^{\beta}}\right),
$$


which is all we need. The proof works analogously for the integrals on the right side of the interval $\left[-x^{\alpha}, x^{\alpha}\right]$.

Theorem 1.

$$
\tilde{\phi}(x)=e^{w(\sigma, x)}\left(2 \sqrt{\frac{\pi}{h(x)}} \sum_{n=0}^{\infty} e^{-\frac{\pi^{2}(2 n+1)^{2}}{4 h(x)}} \cos ((2 n+1) \pi \sigma)+O\left(\frac{1}{x^{\beta}}\right)\right) .
$$

Proof. We use Lemma 3 to obtain

$$
\begin{aligned}
& \tilde{\phi}_{\mathcal{C}_{1}}(x)+\tilde{\phi}_{\mathcal{C}_{2}}(x)= \\
& =\frac{1}{2 \pi i} \int_{-x^{\alpha}}^{x^{\alpha}} \frac{\pi}{\sin (\pi(\sigma+t+i))} e^{w(\sigma+t+i, x)} d t \\
& -\frac{1}{2 \pi i} \int_{-x^{\alpha}}^{x^{\alpha}} \frac{\pi}{\sin (\pi(\sigma+t-i))} e^{w(\sigma+t-i, x)} d t \\
& =e^{w(\sigma, x)+O\left(\frac{1}{x^{\beta}}\right)} \frac{1}{2 \pi i} \int_{-x^{\alpha}}^{x^{\alpha}} \frac{\pi}{\sin (\pi(\sigma+t+i))} e^{-h(x) t^{2}} d t \\
& -e^{w(\sigma, x)+O\left(\frac{1}{x^{\beta}}\right)} \frac{1}{2 \pi i} \int_{-x^{\alpha}}^{x^{\alpha}} \frac{\pi}{\sin (\pi(\sigma+t-i))} e^{-h(x) t^{2}} d t \\
& =e^{w(\sigma, x)} \sum_{n \in\left[\sigma-x^{\alpha}, \sigma+x^{\alpha}\right]}(-1)^{n} e^{-h(x)(n-\sigma)^{2}}+e^{w(\sigma, x)} O\left(\frac{1}{x^{\beta}}\right),
\end{aligned}
$$

using the residue theorem. Furthermore, we have

$$
\begin{aligned}
& \sum_{\substack{n \in\left(\sigma-x^{\alpha}, \sigma+x^{\alpha}\right) \\
n \in \mathbb{Z}}}(-1)^{n} e^{-h(x)(n-\sigma)^{2}}=\sum_{n \in \mathbb{Z}}(-1)^{n} e^{-h(x)(n-\sigma)^{2}}+O\left(\frac{1}{x^{\beta}}\right) \\
= & 2 \sqrt{\frac{\pi}{h(x)}} \sum_{n=0}^{\infty} e^{-\frac{\pi^{2}(2 n+1)^{2}}{4 h(x)}} \cos ((2 n+1) \pi \sigma)+O\left(\frac{1}{x^{\beta}}\right),
\end{aligned}
$$

by Fourier expansion. We finish the proof with Lemma 5.

Let $1<\delta<\beta$ and

$$
j(k)=k \log 2+\log k-\log 2+\frac{d}{k^{\delta}} \text { for an arbitrary real } d .
$$

\section{Lemma 6.}

$$
\pi \sigma(j(k))=k \pi+\frac{\pi}{2}-\frac{d \pi}{\log 2} \frac{1}{k^{\delta}}+O\left(\frac{1}{k^{\beta}}\right) .
$$

Proof. We substitute

$$
(\log 2) k+\frac{\log k-\log 2+\frac{d}{k^{\delta}}}{(\log 2) k}+O\left(\left(\frac{\log k}{k}\right)^{2}\right)
$$


for $\log (j(k))$ and

$$
\frac{1}{(\log 2) k}+O\left(\frac{\log k}{k^{2}}\right)
$$

for $1 / j(k)$. The proof ends after some simplifications.

Theorem 2. There is an integer $K$ such that for all $\epsilon>0$

$$
z_{K+k}=(k+1) 2^{k}+o\left(\frac{2^{k}}{(k+1)^{1-\epsilon}}\right) .
$$

Remark. From the theorem it follows immediately that $z_{k}=k 2^{k}+O\left(2^{k}\right)$. Numerical computations (see Table 1) support the conjecture $K=0$, which is indeed Robinson's conjecture.

Proof. First we choose $0<\alpha<\min \left\{\frac{\epsilon}{3}, \frac{1}{3}\right\}$ and $\beta=2-3 \alpha$, i.e., the constants of Lemma 3 , and $2-\epsilon<\delta<\beta$. By Theorem 1

$$
\tilde{\phi}(x)=e^{w(\sigma, x)}\left(2 \sqrt{\frac{\pi}{h(x)}} f(\sigma)+O\left(\frac{1}{x^{\beta}}\right)\right),
$$

where

$$
f(\sigma)=\sum_{n=0}^{\infty} e^{-\frac{\pi^{2}(2 n+1)^{2}}{4 h(x)}} \cos ((2 n+1) \pi \sigma) .
$$

Let $R_{k}=\left(k \log 2+\log k-\log 2-\frac{1}{k^{\delta}}, k \log 2+\log k-\log 2+\frac{1}{k^{\delta}}\right)$. Lemma 6 guarantees that there exists an $N_{1}$ such that a zero of $f(\sigma(x))$ lies in $R_{k}$, for $k \geq N_{1}$, since a zero of $\cos (\pi \sigma(x))$ is also a zero of $\cos ((2 n+1) \pi \sigma(x))$ for all $n \geq 0$. We have

$$
\left|f^{\prime}(\sigma)\right| \geq e^{-\frac{\pi^{2}}{4 h(x)}}|\sin (\pi \sigma)|-\pi\left|\sum_{n=1}^{\infty}(2 n+1) e^{-\frac{\pi^{2}(2 n+1)^{2}}{4 h(x)}} \sin ((2 n+1) \pi \sigma)\right| .
$$

We notice that

$$
c_{2}=0.34 \leq h(x) \leq 0.42=c_{1}
$$

for $x \geq 10$. Now, we estimate

$$
\begin{aligned}
& \left|\sum_{n=1}^{\infty}(2 n+1) e^{-\frac{\pi^{2}(2 n+1)^{2}}{4 h(x)}} \sin ((2 n+1) \pi \sigma)\right| \\
& \leq \sum_{n=1}^{\infty}(2 n+1) e^{-\frac{\pi^{2}(2 n+1)^{2}}{4 c_{1}}} \leq e^{-6 c_{3}} \sum_{n=1}^{\infty}(2 n+1) e^{-c_{3}(2 n+1)} \leq e^{-6 c_{3}} \frac{e^{-c_{3}}}{\left(1-e^{-c_{3}}\right)^{2}}
\end{aligned}
$$

where $c_{3}=\frac{\pi^{2}}{4 c_{1}}$. Therefore,

$$
\left|f^{\prime}(\sigma)\right| \geq e^{-\frac{\pi^{2}}{4 c_{2}}}|\sin (\pi \sigma)|-e^{-6 c_{3}} \frac{e^{-c_{3}}}{\left(1-e^{-c_{3}}\right)^{2}}>0.0007,
$$


if $\pi \sigma \in\left[k \pi+\frac{\pi}{2}-0.1, k \pi+\frac{\pi}{2}+0.1\right]$. With the mean value theorem and Lemma 6 we obtain that

$$
\left|f\left(k \log 2+\log k-\log 2 \pm \frac{1}{k^{\delta}}\right)\right| \geq \frac{c}{k^{\delta}}+O\left(\frac{1}{k^{\beta}}\right),
$$

where $c>0$. Thus, we observe that there exists an $N$ such that a zero of $f(x)+O\left(\frac{1}{x^{\beta}}\right)$, which is also a zero of $\tilde{\phi}(x)$, is contained in $R_{k}$, for all $k \geq N$. Let $k \geq N$ and $x_{k}$ be the zero of $\tilde{\phi}$ which lies in in $R_{k}$. As Robinson stated in [3, p. 258] we also have $z_{i}>2 z_{i-1}$, for all $i \geq 1$. This means that there can be no zero of $\tilde{\phi}$ between $x_{k}$ and $x_{k+1}$. Since otherwise $x_{k+1}-x_{k}>2 \log 2$, which is impossible, because we showed that $x_{k+1}-x_{k} \leq(k+1) \log 2+\log (k+1)-\log 2+\frac{1}{(k+1)^{\delta}}-\left(k \log 2+\log k-\log 2-\frac{1}{k^{\delta}}\right)=$ $\log 2+\log \left(\frac{k+1}{k}\right)+\frac{1}{(k+1)^{\delta}}+\frac{1}{k^{\delta}}$. Let $M$ be the index of the zero which corresponds to the zero of $\tilde{\phi}(x)$ which lies in $R_{N}$, i.e., $\log z_{M} \in R_{N}$. Then $\log z_{M+i} \in R_{N+i}$, for all $i \geq 1$, and therefore $\log z_{K+k} \in R_{k}$, for $k \geq N$, where $K=M-N$. This means that

$$
\log z_{K+k}=(k+1) \log 2+\log (k+1)-\log 2+O\left(\frac{1}{(k+1)^{\delta}}\right),
$$

or in other words

$$
\begin{aligned}
z_{K+k} & =(k+1) 2^{k} e^{O\left(\frac{1}{(k+1)^{\delta}}\right)}=(k+1) 2^{k}+O\left(\frac{2^{k}}{(k+1)^{\delta-1}}\right) \\
& =(k+1) 2^{k}+o\left(\frac{2^{k}}{(k+1)^{1-\epsilon}}\right),
\end{aligned}
$$

since $1-\epsilon<\delta-1$, finishing the proof.

\section{REFERENCES}

[1] N. G. De Bruijn, Asymptotic methods in analysis, (North-Holland Publishing Co.-Amsterdam, 1958)

[2] P. Henrici, Applied and Computational Complex Analysis, (Pure and Applied Mathematics, A Wiley Interscience Series of Texts, Monographs \& Tracts, John Wiley \& Sons, 1977)

[3] R.W. Robinson, Counting labeled acyclic digraphs, in: F. Harary, ed., New Directions in the Theory of Graphs (Academic Press, New York, 1973) 239-279.

[4] R.W. Robinson, Counting unlabeled acyclic digraphs, Lect. Notes Math. 622 (1977) 28-43.

[5] R. P. Stanley, Acyclic orientations of graphs, Discrete Mathematics, 5, No. 2 (1973), 171-178.

Institut für Mathematik A, Technische Universität Graz, Steyrergasse 30, 8010 Graz, AUstria

E-mail address: peter.grabner@tugraz.at

E-mail address: steinsky@finanz.math.tu-graz.ac.at 